\title{
Tip-induced distortions in STM imaging of carbon nanotubes
}

\author{
Heinz Hövel ${ }^{1 \mathrm{a}}$, Mario De Menech ${ }^{2,3}$, Mirko Bödecker ${ }^{1}$, Christian Rettig $^{1}$, Ulf Saalmann ${ }^{2}$, and Martin E. Garcia ${ }^{3}$ \\ 1 Experimentelle Physik I, Universität Dortmund, 44221 Dortmund, Germany \\ 2 Max-Planck-Institut für Physik komplexer Systeme, Nöthnitzer Str. 38, 01187 Dresden, Germany \\ 3 Theoretische Physik, FB 18 and Center for Interdisciplinary Nanostructure Science \\ and Technology, Universität Kassel, Heinrich-Plett-Str. 40, 34132 Kassel, Germany
}

\begin{abstract}
By means of STM measurements and fully self-consistent transport calculations we analyze how STM trajectories for the mapping of nanostructures on surfaces are affected by the atomic structure of the tip. For the particular case of carbon nanotubes we show that considerable distortions of the STM trajectory with respect to the actual structure, position and diameter of the nanotube can occur for certain tip geometries. Comparison between theory and experiment can allow to characterize and correct these distortions.
\end{abstract}

PACS. 68.37.Ef Scanning tunneling microscopy - 73.63.-b Electronic transport in nanoscale materials and structures $-61.48 .+\mathrm{c}$ Fullerenes and fullerene-related materials

\section{Introduction}

The interpretation of STM images of nanometer-sized objects is far from being a trivial task, because the size and shape of these objects can be significantly distorted due to the influence of the shape of the STM tip and the threedimensional trajectory of the scan process. This problem was discussed for STM imaging of clusters [1] where it leads to a significant inflation of the measured cluster width. A similar enhancement of the width also occurs for

\footnotetext{
a e-mail: hoevel@physik.uni-dortmund.de
}

the STM imaging of nanotubes. As a consequence, the interpretation of the atomic structure of nanotubes in STM images becomes cumbersome. A geometric model based on the assumption of a constant tunneling distance and the radial projection of the atomic structure was used within the simulation of STM images in a tight-binding computation [2]. The existence of an expansion of the image scale perpendicular to the tube axis was confirmed in experimental data [3] and included in the quantitative analysis of the nanotube lattice orientation with respect to the sub- 
strate [4]. However, in Ref. [4] the expansion was used only as a parameter for a local fit of the nanotube lattice. Here, we have carried out a systematic analysis of STM measurements of this effect on supported carbon nanotubes. In addition, we have performed accurate microscopic calculations based on non-equilibrium Greens function. We will discuss the validity of simple geometric considerations on the basis of the experimental data obtained and a detailed theoretical modeling of the STM process.

\section{Experimental observations}

For the experimental data we focus on two data sets out of different measurements reported before $[3,4]$. In Fig. 1a we show the STM image of a carbon nanotube on a graphite substrate. Each horizontal line, parallel to the tube axis, is set to the same mean height for a better visibility of the atomic structure. As already mentioned in Ref. [3], a reasonable fit to the atomic tube structure is obtained if we construct an ideal graphite lattice which fits the simultaneously imaged graphite substrate and expand it by a factor of about 1.6 perpendicular to the tube axis. This indicates an orientation of the tubes given by an alignment of the tube graphene structure with the lattice of the graphite substrate. Later on, the analysis of the tube lattice orientation was refined [4] using a technique for evaluating the mutual orientation which uses the measured lattice vectors of the graphite surface as a reference for distortion and drift effects in the STM image. This not only confirmed the alignment of the nanotube lattice structure to the structure of the graphite substrate, but also gave a better value for the factor by which the nanotube lattice is expanded perpendicularly to the tube axis. For the tube in Fig. 1 we obtained $z \approx 1.4$. For other tubes the value of $z$ was always in the range between 1.35 and 1.8 .
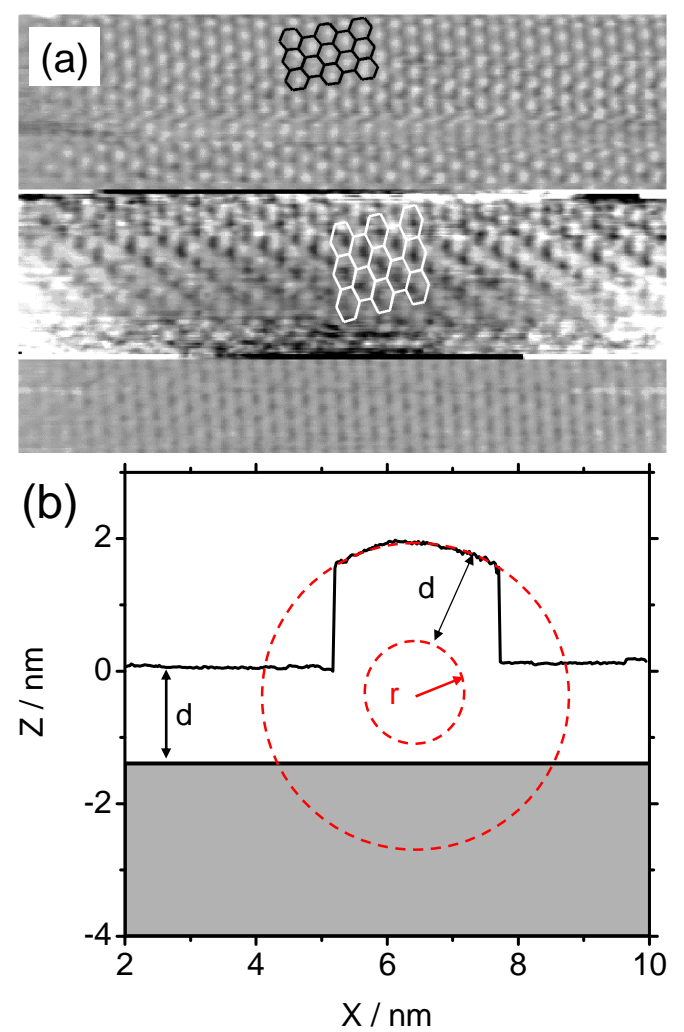

Fig. 1. (a) STM image of a carbon nanotube on HOPG. Two Honeycomb lattices are shown: one fitted to the HOPG substrate (black) and the other expanded perpendicularly to the nanotube axis to fit its atomic structure (white). (b) An experimental line profile perpendicular to the tube axis (black/solid) is fitted assuming that the tip is moving on a circle with radius $d+r$ centered to the tube with radius $r$ (red/dashed). The tunneling distance $d$ is assumed to be equal on the substrate and on top of the tube.

In Fig. 1b an STM line profile perpendicular to the tube is shown with equal $x$ - and $z$-scaling. If we adopt 
the geometric model with a constant tunneling distance [2] and fit a circle to the top-curvature of the line profile, we get a circle diameter $2 R=2 r+2 d$ of more than $4 \mathrm{~nm}$, with $2 r$ the nanotube diameter and $d$ the distance between tunneling tip and nanotube. We first assume that the tunneling distance is equal for the graphite substrate and the nanotube, and then that the distance between tube and substrate is smaller than $0.35 \mathrm{~nm}$ (the lattice plane distance of graphite), due to the expected deformation of the tube at the substrate interface [5]. The tube diameter given by total height of the line profile minus the distance tube-substrate is then $2 r \approx 1.7 \mathrm{~nm}$. However, two problems arises for the application of the simple geometric model. Firstly, the tunneling distance $d$ is significantly larger than typical distances of about $0.5 \mathrm{~nm}$. Since the tunneling current decreases by one order of magnitude for each $\Delta d \approx 0.1 \mathrm{~nm}$, this distance seems unrealistically large for the rather typical tunneling parameters $U=0.3 \mathrm{~V}$ and $I=0.35 \mathrm{nA}$. Secondly, the geometric model $[2]$ predicts $z=1+d / r$ which would give $z \approx 2.8$, i. e. significantly larger than the observed expansion factor of $z \approx 1.4$.

The steep increase at the tube edges may be due to the characteristics of the STM control loop; but also a mechanical deformation of the tube can not be excluded, which allows an approach much closer than the one for a static position of the tube before the tip retracts. A mechanical instability would also explain that the STM image is often very noisy at the edges of the tube while the image shows atomic resolution on the substrate and on the top of the tube.



Fig. 2. (a) STM image of a carbon nanotube on HOPG. One can observe two different tube diameters (left and right of the junction), which shows up as a small extra hillock. (b) Experimental line profiles perpendicular to the tube axis for the left part (black/thin solid) and the right part (red/thick solid). The tip trajectories are fitted with circles of radius $r+d$ centered to the tube with radius $r$ for the left part (black/thin dashed) and for the right part (red/thick dashed). The tunneling distance $d>2 \mathrm{~nm}$ is unrealistically large.

The increased tunneling voltage of $U=1.0 \mathrm{~V}$ and the decreased tunneling current in comparison to Fig. 1 may be the reason why the image in Fig. 2 is "softer" with less steep edges of the tube. In Fig. 2a the three-dimensional 
image suggests that one sees a direct image of the nanotube shape, but this is only due to the expanded $z$-scale, clearly shown in Fig. $2 \mathrm{~b}$ by the line profiles crossing the two different tube parts. For the interpretation of the data using the model of constant radial tunneling distance $d$ we omitted for simplicity in Fig. 2b the nanotube-substrate distance, since $d \gg r$. This is also the reason why the curvature is similar for both line profiles, despite the different tube height for the two parts. Here $d$ is even larger than in Fig. 1b, amounting to more than $2 \mathrm{~nm}$ - which is not realistic at all. This large $d$ for the small tubes of heights less than $1 \mathrm{~nm}$ would correspond to an expansion factor $z>5$, which is again much larger than the $z \approx 1.75$ extracted from the experiment using the procedure as explained in Ref. [4].

These two experimental examples clearly show that the model of constant tunneling distance $d$ and radial projection of the atomic structure of the tube does not fit to the experiment. This may be partly due to the real tip shape in STM, which is in general far apart from the point source assumed in the geometrical model [2]. By modeling the tip trajectory in a more realistic calculation even a rather sharp tip leads to deviations from the simple geometric model as it will be shown and discussed in the following section.

\section{Microscopic calculations}

We have performed microscopic calculations of the STM imaging procedure for finite nanotubes. These are based on a non-equilibrium Green's function description of the electron transport from the tip, mounted at the upper electrode, through the tube to the supporting surface. The interactions with the electrode and the surface are taken into account via tunneling self-energies [6]. The tube and the tip, however, are treated in a fully atomistic description, which is of crucial importance with respect to the questions discussed above. We employ a self-consistent tight-binding model which is parameterized from densityfunctional calculations; atomic charge fluctuations (transfer and polarization) are taken into account [7]. The procedure for calculating equilibrium properties, like the charge transfer to or from the nanotube, and transport properties, like the current through the nanotube, is described elsewhere [8].

Our microscopic approach does not allow to treat tubes of diameters and lengths similar to those presented in Sect. 2. Instead, we performed investigations of $(4,4)$ tubes [9] with a length of 20 repeat units, placed on a $\mathrm{Au}(111)$ surface [10]. Note that such tube sizes with 160 carbon atoms require extensive numerical calculations. We employed two different tips with 10 atoms arranged in a pyramidal shape simulating "realistic" tips. The first one is sharp with three layers of 1,3 , and 6 atoms; the second one is blunt with two layers of 3 and 7 atoms. Figure 3 compares the tip trajectories of the two tips for the same nanotube. The trajectories, i. e. the tip heights, are shown as a function of the tip offset from the tube axis. Calculations for different positions along the tube axis lead to a spread of the heights for a given transversal offset as can be seen in Fig. 3. More importantly, the figure also 

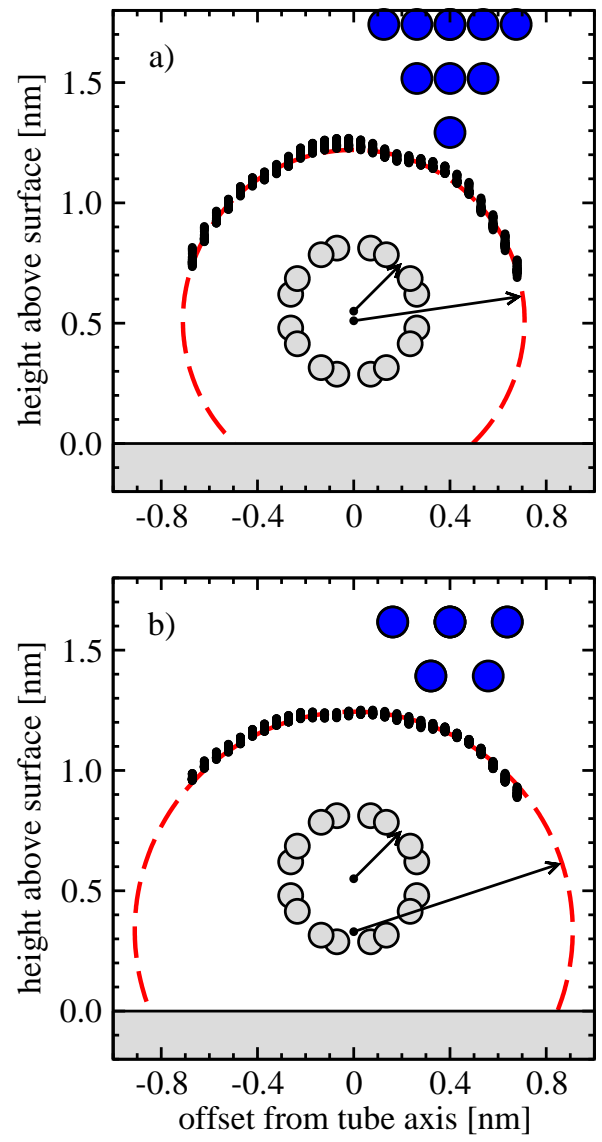

Fig. 3. Calculated STM tip trajectories (small black circles) for a $(4,4)$ nanotube (indicated by grey-filled/light circles) using two different tip shapes (blue-filled/dark circles): a) sharp tip, b) blunt tip. The spread in the heights for a given transversal offset from the tube axis results from different longitudinal positions of the tip. Note that trajectories can be fitted in both cases by a circle (red/thick dashed line).

shows that - independently of the tip shape under consideration — the trajectories can be nicely fitted by circles. Note, however, that the radii $R$ of these fitting circles differ, $R_{\text {sharp }}=0.7 \mathrm{~nm}$ vs. $R_{\text {blunt }}=0.9 \mathrm{~nm}$. As can be seen in Fig. 3a, there is a tiny shift of the center of the circle with respect to the tube center for the sharp tip. Neglecting it one gets back to the simple geometric model proposed earlier [2]. On the other hand, cf. Fig. 3b, there is a clear shift by about $0.2 \mathrm{~nm}$ for the blunt tip. That means, that the experimentally observed circular line profiles do not allow to conclude a constant distance of tip and tube. Rather the distance from the tube to the closest atom of the tip — which may change for different offsets — remains constant. Indeed, as observed in Fig. 3 we obtain centrally over the tube for both cases a similar tunneling distance of $d \approx 0.4 \mathrm{~nm}$.

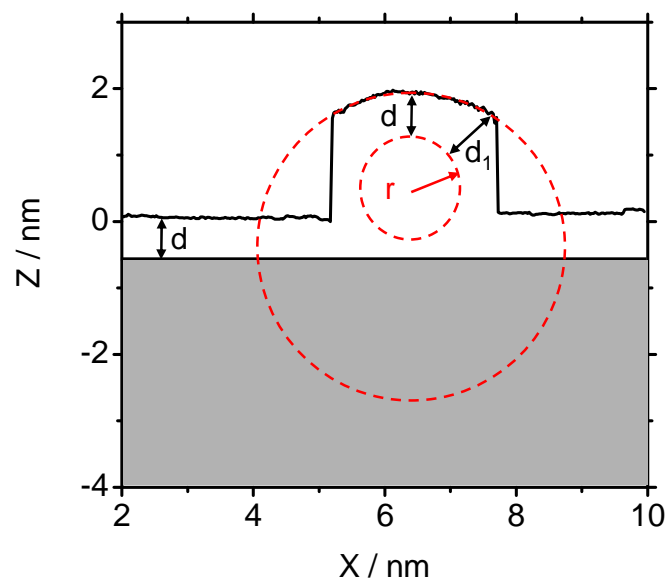

Fig. 4. Line profile from Fig. 1b (black/solid) fitted with a realistic tunneling distance of $d \approx 0.5 \mathrm{~nm}$ on the substrate and on top of the tube. The center of the circle describing the tip trajectory is shifted with respect to the tube center (red/dashed). At the edge of the tube image the distance between the tip trajectory and the tube is increased to $d_{1} \approx 0.8 \mathrm{~nm}$.

With these results of the microscopic calculation we now may modify the interpretation of the STM trajectory in Fig. 1b. If we allow for a shift of the circle describing the tip trajectory we are able to reduce the tunneling distance to the realistic value of $d=0.5 \mathrm{~nm}$ on the substrate and on top of the tube as shown in Fig. 4. In this geometry the expansion of the lattice perpendicular to the tube axis is still 
given by $z=1+d / r$ for the local radial projection. The distance of the tip trajectory and the tube varies between $d \approx 0.5$ and $d_{1} \approx 0.8 \mathrm{~nm}$, cf. Fig. 4 , which corresponds for $r=0.85 \mathrm{~nm}$ to $z \approx 1.6$ or $z_{1} \approx 1.9$ respectively. This is in better agreement with the experimental results. However, since different tip atoms may contribute to imaging the atomic structure of the tube, a more detailed modeling for the distortion parameter $z$ may be needed.
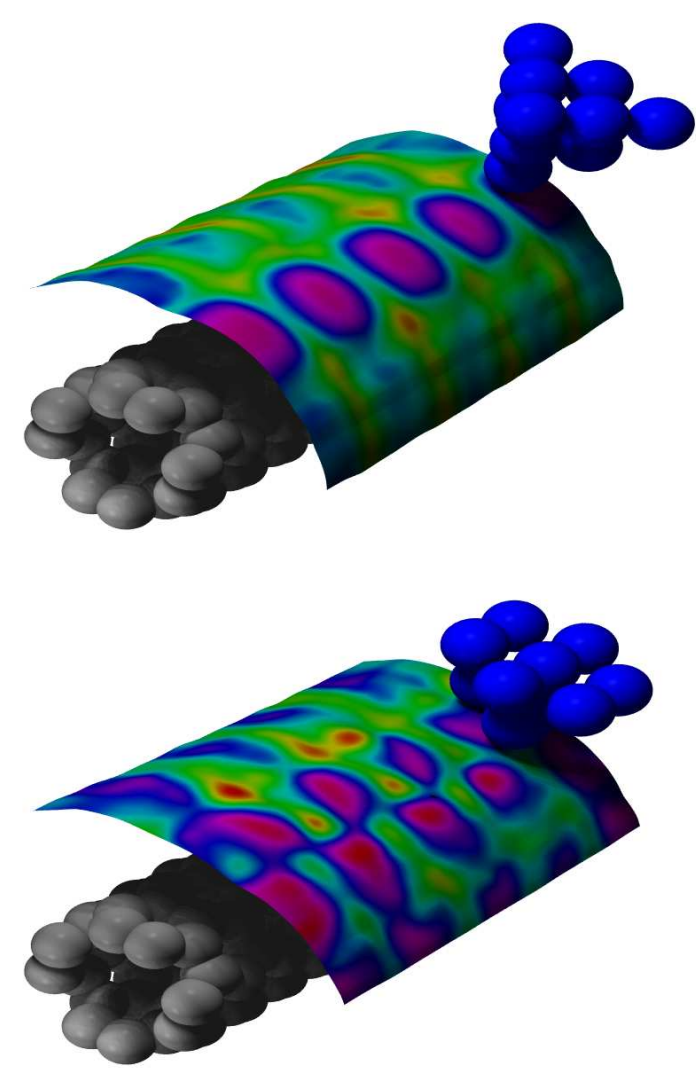

Fig. 5. Conductance $\mathrm{d} I / \mathrm{d} V$ through a $(4,4)$ carbon nanotube from the two STM tips used for Fig. 3 shown as colored contour plot projected onto the constant-current surface. The upper electrode and the supporting surface are not shown.

The topography of the $(4,4)$ tube considered in the calculations does not give useful information. Because of the small radius of the tube, the fine structure of the atomic structure does not show up clearly, since the displacement of the tip in the vertical direction due to the radius is dominating the topography. Therefore, we present STS or energy-resolved conductance maps, which do not suffer from the strong curvature of the tube. Figure 5 shows for both tip shapes conductance maps $\mathrm{d} I / \mathrm{d} V(x, y)$ at a bias of $0.6 \mathrm{~V}$ projected onto the constant-current surface. In the calculations, the tip was moved on a three-dimensional finite-element mesh with a grid spacing of $0.05 \mathrm{~nm}$. At each grid point the current and the conductance were computed, and the constant-current isosurface was obtained by interpolation.

In both cases periodic patterns along the tube axis from standing waves in the finite tubes are visible. Obviously, their wavelength is larger than the width $0.123 \mathrm{~nm}$ of one repeat unit. The Fourier transformations of such patterns for various bias values may be used to construct the dispersion relation of the considered tube [11]. More important here, the mapping clearly allows for the characterization of the STM tip. For the "blunt" tip, with an equilateral triangle at the bottom, we note a dichroism, i. e. repeated conductance peaks due to different end atoms of the tip, which has been observed before in "abnormal maps" for imaging of $\mathrm{C}_{60}$ molecules [8]. It is exactly this change of the current-carrying atom which widens the tip trajectory as shown above in Fig. 3. For yet broader tips we expect even more involved STS maps. 


\section{Conclusions}

We have shown that the simple geometric model of constant tunneling distance and radial projection of the atomic structure, which was suggested to take into account for the distortions observed in STM images of carbon nanotubes, does not fit to the experimental results. With detailed microscopic calculations of the STM imaging procedure for different ("sharp" and "blunt") tip geometries we arrived at a more realistic model in which the distance between the tip trajectory and the nanotube is no longer constant for a "blunt" tip. Nevertheless, the transversal motion of the STM tip occurs on a circle with the circle center shifted with respect to the tube center. This finding is in very good agreement with the experiment.

The topics discussed in this work will be important not only for nanotubes but also for STM imaging of other nanometer sized objects on surfaces as, e.g., deposited clusters [11].

\section{Acknowledgments}

We gratefully acknowledge financial support by the Deutsche Forschungsgemeinschaft through the priority program SPP 1153 "Clusters in contact with surfaces: Electronic structure and magnetism". We thank Anatole Kenfack for carefully reading the manuscript.
2. V. Meunier and Ph. Lambin, Phys. Rev. Lett. 81, 5588 (1998).

3. H. Hövel, M. Bödecker, B. Grimm, and C. Rettig, J. Appl. Phys. 92, 771 (2002).

4. C. Rettig, M. Bödecker, and H. Hövel, J. Phys. D 36, 818 (2003).

5. T. Hertel, R. E. Walkup, and P. Avouris, Phys. Rev. B 58, 13870 (1998).

6. S. Datta, Electronic tranpsort in mesoscopic systems. Cambridge University Press 1995.

7. M. Elstner et al., Phys. Rev. B 58, 7260 (1998).

8. M. De Menech, U. Saalmann, and M. E. Garcia, Phys. Rev. B 73, 155407 (2006).

9. R. Saito, M. S. Dresselhaus, and G. Dresselhaus (eds.) Physical properties of carbon nanotubes. Imperial College Press 1998.

10. We believe that using a Au surface does not have any measurable influence on the discussed tip effect.

11. M. De Menech, U. Saalmann, and M. E. Garcia, to be published.

\section{References}

1. H. Hövel, Appl. Phys. A 72, 295 (2001). 